\title{
Exposición involuntaria al humo de tabaco en lugares públicos de la Ciudad de México
}

\author{
Tonatiuh Barrientos-Gutiérrez, MD, M en C, ${ }^{(1,2)}$ Raydel Valdés-Salgado, MSc, ${ }^{(3)}$ \\ Luz Myriam Reynales-Shigematsu, MD, M en C, Dra en C, ${ }^{(2)}$ Ana Navas-Acien, MD, PhD, ${ }^{(3)}$ \\ Eduardo Lazcano-Ponce, MD, M en C, Dr en C. ${ }^{(2)}$
}

\section{Barrientos-Gutiérrez T,Valdés-Salgado $R$ Reynales-Shigematsu LM, Navas-Acien A, Lazcano-Ponce E. Exposición involuntaria al humo de tabaco en lugares públicos de la Ciudad de México. Salud Publica Mex 2007;49 supl 2:S205-S2 I2.}

\section{Resumen}

Objetivo. La legislación mexicana considera como espacios libres de humo de tabaco a muchos lugares públicos. Sin embargo, no existen evaluaciones de la exposición a humo de tabaco ambiental que permitan medir el cumplimiento de las leyes ni identificar espacios de oportunidad para el control de la exposición. El presente estudio tiene como objetivo cuantificar los niveles de nicotina ambiental en lugares públicos de la Ciudad de México. Material y métodos. Utilizando un protocolo común a otros países de América Latina, durante marzo de 2004 se colocaron monitores de difusión pasiva de nicotina en un hospital, dos escuelas, un edificio de oficinas públicas, un aeropuerto, siete restaurantes y tres bares de la Ciudad de México. Los monitores fueron analizados en la Universidad Johns Hopkins, donde la nicotina fue extraída y cuantificada usando cromatografía de gases con detección selectiva de nitrógeno. La concentración mediana de nicotina ambiental fue estimada por tipo de lugar y por área de restricción para fumar. Resultados. Se detectó nicotina ambiental en todos los lugares públicos. Las mayores concentraciones se observaron en bares $\left(6.01 \mu \mathrm{g} / \mathrm{m}^{3}\right)$, restaurantes $\left(0.69 \mu \mathrm{g} / \mathrm{m}^{3}\right)$, aeropuerto $\left(0.21 \mathrm{\mu g} / \mathrm{m}^{3}\right)$ y oficinas públicas $\left(0.10 \mu \mathrm{g} / \mathrm{m}^{3}\right)$. Los grados de exposición fueron similares a los observados en otras ciudades de América Latina. Conclusiones. Las concentraciones de nicotina en el aeropuerto y las oficinas públicas
Barrientos-Gutiérrez T,Valdés-Salgado $R$,

Reynales-Shigematsu LM, Navas-Acien A, Lazcano-Ponce E.

Involuntary exposure to tobacco smoke

in public places in Mexico City.

Salud Publica Mex 2007;49 suppl 2:S205-S2I2.

\begin{abstract}
Objective. Mexican legislation considers many public places as smoke-free spaces. However, no environmental tobacco smoke exposure assessment studies exist to evaluate compliance with governmental regulations and to identify opportunities for tobacco control.The main objective of this study is to quantify airborne nicotine concentrations in public places in Mexico City. Material and Methods. During March 2004, nicotine passive monitors were allocated in a hospital, two schools, a public office building, an airport, seven restaurants, and three bars following a common protocol applied in other Latin American countries. Monitors were analyzed at the Johns Hopkins University, extracting the nicotine and quantifying it using nitrogen selective gas chromatography. Median concentrations of environmental nicotine were estimated by type of place and smoking restriction. Results. Airborne nicotine was detected in all public places. The highest concentrations were observed in bars $\left(6.01 \mu \mathrm{g} / \mathrm{m}^{3}\right)$, restaurants $\left(0.69 \mu \mathrm{g} / \mathrm{m}^{3}\right)$, airport $\left(0.21 \mu \mathrm{g} / \mathrm{m}^{3}\right)$, and public offices $\left(0.10 \mu \mathrm{g} / \mathrm{m}^{3}\right)$. Nicotine exposure concentrations are similar to those previously observed in other capital cities of Latin America. Conclusion. Nicotine levels in the airport and public offices reflect the lack of compliance with mandatory non-smoking official regulations in Mexico. High nicotine concentrations in bars and restaurants provide evidence for
\end{abstract}

(I) School of Public Health, The University of Texas, EUA.

(2) Instituto Nacional de Salud Pública. México.

(3) Department of Epidemiology, Johns Hopkins Bloomberg School of Public Health. EUA.

Fecha de recibido: 4 de abril de 2007• Fecha de aprobado: 30 de abril de 2007

Solicitud de sobretiros: Mtro. Tonatiuh Barrientos-Gutiérrez. The Southwest Center for Occupational and Environmental Health. The University of Texas, School of Public Health at Houston. 1200 Hermann Pressler,W-I004-I Houston, Texas, 77030, USA.

Correo electrónico:Tonatiuh.Barrientos@uth.tmc.edu 
reflejan la falta de cumplimiento con la legislación vigente en México. Las concentraciones de nicotina registradas en bares y restaurantes evidencian la necesidad de impulsar medidas legislativas que establezcan a estos sitios como lugares públicos y de trabajo libres de humo de tabaco.

Palabras clave: humo de tabaco; nicotina; lugar público; lugar de trabajo; vigilancia; México the need to advance smoke-free legislative action in these public and work places.

Key words: tobacco smoke; nicotine; public places; work places; surveillance; Mexico
$V^{a t s}$ asta evidencia epidemiológica y toxicológica ha demostrado que la exposición al humo de tabaco ambiental causa daños graves a la salud de los no fumadores, asociándosele con cáncer de pulmón, enfermedades cardiovasculares, respiratorias y reproductivas, entre otras. ${ }^{1} \mathrm{El}$ humo de tabaco contiene más de 250 compuestos tóxicos o carcinógenos y carece de valores seguros de exposición, por lo que cualquier grado de exposición es susceptible de provocar daño. La exposición ocurre en lugares donde se fuma, por lo que la solución más efectiva y barata es prohibir fumar en lugares cerrados. El costo humano y económico de las enfermedades derivadas de la exposición involuntaria a humo de tabaco es un problema mundial. En respuesta, se han enunciado disposiciones internacionales para garantizar que los lugares públicos estén libres de humo de tabaco; tal es el caso del Convenio Marco para el Control del Tabaco de la Organización Mundial para la Salud, el cual ha sido firmado y ratificado por más de 160 pa ses, incluido México en el año 2004. ${ }^{2}$

Numerosos pa ses y regiones de todo el mundo han desarrollado leyes completas para controlar este problema, incluidos Irlanda ${ }^{3}$ e Italia ${ }^{4}$ en Europa, y California ${ }^{5}$ y Nueva York ${ }^{6}$ en Estados Unidos. En América Latina, solamente Uruguay y Puerto Rico tienen leyes integrales que garantizan ambientes libres de humo de tabaco en todos los lugares públicos y centros de trabajo del pa s, incluidos bares y restaurantes. ${ }^{7,8}$ En la Ciudad de Buenos Aires, Argentina, se ha impulsado la prohibición total de consumo de tabaco en la mayo$\mathrm{r}$ a de los lugares públicos. ${ }^{9}$ En México, el cap tulo XI, art culo 277 bis, de la Ley General de Salud establece la normatividad para la creación de lugares libres de humo de tabaco en los servicios de atención médica de la Secretar a de Salud, auditorios, aulas y zonas de peligro para la seguridad laboral y colectiva, as como en los sitios de trabajo de ambiente cerrado, oficinas federales, instituciones educativas y centros de transporte de la República Mexicana. ${ }^{10}$

No obstante, a pesar de la existencia de medidas legislativas para el control del consumo de tabaco en lugares públicos, los grados de exposición siguen siendo graves en casi todos los pa ses latinoamericanos y evidencian la falta de adecuación o cumplimiento de las leyes imperantes. ${ }^{11}$ En México no existen sistemas de vigilancia continua de la exposición al humo de tabaco ambiental, por lo que los niveles de exposición involuntaria en lugares públicos no han sido evaluados de manera sistemática. La vigilancia continua de estos grados de exposición en México es necesaria para evaluar el cumplimiento de las disposiciones oficiales e identificar áreas de oportunidad para el desarrollo de medidas preventivas y legislativas eficaces. El presente estudio tiene como objetivo evaluar la exposición a humo de tabaco en hospitales, escuelas, aeropuertos, bares y restaurantes de la Ciudad de México mediante un protocolo común a otros pa ses latinoamericanos. ${ }^{11}$

\section{Material y métodos \\ Lugares en estudio}

Los lugares públicos elegidos por conveniencia para el monitoreo de nicotina ambiental fueron una institución de salud (hospital de tercer nivel de atención), dos escuelas secundarias públicas, un edificio de oficinas públicas, un aeropuerto, siete restaurantes y tres bares; los criterios de inclusión fueron pertenecer a cualquiera de las categor as arriba descritas, localizarse en la Ciudad de México y estar dispuesto a proveer un consentimiento informado para efectuar la investigación. De manera inicial, el equipo de investigación visitó los lugares seleccionados, donde realizó un registro de las caracter sticas de cada lugar y espacio de monitoreo, el tipo de restricción (área de fumar o no fumar), el tiempo de monitoreo y las condiciones de ocupación (número de personas) y ventilación de cada espacio. Asimismo, se obtuvieron los permisos oficiales para llevar a cabo el monitoreo con los representantes legales de las instituciones y lugares en estudio. El protocolo de estudio fue aprobado por el comité de ética de la Universidad Johns Hopkins y del Instituto Nacional de Salud Pública. 


\section{Evaluación de la exposición a humo de tabaco ambiental}

Durante la evaluación efectuada en marzo de 2004 se utilizaron monitores de difusión pasiva para cuantificar la nicotina ambiental en fase de vapor, que es uno de los compuestos derivados del humo de tabaco preferidos para el monitoreo dada su alta especificidad y la disponibilidad de métodos para su captura y cuantificación. ${ }^{1}$ Los monitores de difusión pasiva, diseñados por $\mathrm{K}$. Hammond para la medición de nicotina en fase de vapor, son casetes de plástico de $34 \mathrm{~mm}$ que contienen un filtro impregnado de bisulfato de sodio, una sustancia con alta afinidad a la nicotina. La cantidad de aire que pasa por los monitores de difusión pasiva por minuto, o tasa de flujo, ha sido estimada en $24 \mathrm{ml} / \mathrm{min}^{12}$ Los monitores de difusión pasiva han sido validados en condiciones experimentales ${ }^{12,13}$ y utilizados ampliamente en estudios de monitoreo ambiental.11,14,15

Para esta evaluación se colocaron 100 monitores de acuerdo con la asignación presentada en el cuadro I: 27 en el hospital, 9 en cada escuela, 21 en las oficinas públicas, 14 en el aeropuerto, 2 en cada restaurante (uno en el área de fumar y uno en la de no fumar) y 2 en cada bar. Al mismo tiempo se instalaron 10 filtros duplicados $(10 \%)$ y 10 filtros blancos $(10 \%)$ para efectos de control de calidad y cuantificación de concentraciones $\mathrm{m}$ nimas detectables de nicotina. Los monitores blancos se transportaron, manipularon y analizaron de la misma forma que el resto de los monitores, pero sin exponerlos al aire de los lugares. Dentro de cada lugar, los monitores fueron colocados en un sitio representativo del área que se pretend a medir, de manera habitual, a una distancia de 2 metros o más a partir del suelo.

La duración del monitoreo dependió del nivel de exposición a humo de tabaco ambiental previsto por el equipo de investigación. En restaurantes y bares, lugares con alto potencial para exposición a humo de tabaco, se monitoreó por una semana con visitas diarias para vigilar la permanencia y adecuada colocación de los monitores, la cantidad de ocupantes y las condiciones de ventilación (número de ventanas y puertas abiertas, y sistemas de ventilación mecánica). En el resto de los lugares, el monitoreo se efectuó durante dos semanas con visitas cada dos d as. Una vez cumplido el tiempo establecido, los monitores fueron sellados dentro de los lugares monitoreados y enviados desde el Instituto Nacional de Salud Pública al Laboratorio de la Escuela de Salud Pública de la Universidad Johns Hopkins.

En el laboratorio, la nicotina se extrajo del filtro según un procedimiento estándar ${ }^{12}$ y se analizó mediante cromatograf a gaseosa con detección selectiva de nitrógeno. Las concentraciones ambientales de nicotina para cada filtro se calcularon dividiendo la cantidad de nicotina extra da del filtro entre el volumen de aire muestreado (número de minutos de tiempo de muestreo multiplicado por la tasa de flujo). Las concentraciones m nimas de detección (CMD) de nicotina se calcularon a partir de la curva de regresión y se ajustaron por el periodo de muestreo, con lo cual se obtuvo una CMD de $0.004 \mu \mathrm{g} / \mathrm{m}^{3}$ para el periodo de dos semanas y de $0.009 \mu \mathrm{g} / \mathrm{m}^{3}$ para una semana. Para efectos del análisis, los filtros con concentraciones no detectables de nicotina fueron imputados con el valor de la CMD dividida entre la ra $\mathrm{z}$ cuadrada de 2.

\section{Análisis}

En general y para cada tipo de lugar se utilizaron medidas de tendencia central y dispersión. De forma adicional, se analizaron las concentraciones obtenidas en áreas de fumar y no fumar de los lugares con medidas de restricción expl citas (restaurantes y aeropuertos). Para la descripción de las concentraciones de nicotina ambiental se utilizaron la mediana y el rango intercuart lico (RIC) dado que la nicotina tiende a seguir una distribución log-normal. ${ }^{16}$ Se utilizaron gráficas de caja para representar las distribuciones de nicotina por lugar y tipo de zona (fumar-no fumar). Todos los procedimientos fueron realizados en STATA 8.2.

\section{Resultados}

El cuadro I describe la colocación de monitores, el número de monitores perdidos y el número de monitores debajo de la concentración m nima de detección. De los 100 monitores colocados inicialmente, 12 se perdieron o se dañaron durante el monitoreo. La mayor cantidad de filtros perdidos o dañados ocurrió en las escuelas (30\% de los colocados). De los 88 filtros analizados, 72 (82\%) tuvieron concentraciones por encima de la concentración m nima de detección, considerándose lugares con exposición a humo de tabaco en el ambiente. Casi todos los filtros con resultados por debajo de las concentraciones $\mathrm{m}$ nimas de detección se encontraron en las escuelas, el hospital y las oficinas públicas, considerados, por lo tanto, lugares libres de humo de tabaco. En los bares, restaurantes y el aeropuerto todas las concentraciones de nicotina registradas estuvieron por encima de la concentración m nima de detección.

Las escuelas y el hospital presentaron concentraciones de nicotina ambiental bajas y consistentes (cuadro II y figura 1). Las oficinas públicas y el aeropuerto presentaron valores más altos de nicotina con una gran variabilidad, es decir, algunos de sus espacios presentaban mayores concentraciones de nicotina que 
Cuadro I

Monitores COLOCADOS, PERDIDOS, ANALIZAdOS Y POR DEBAJO DE LA CMD* EN LUGARES PÚBLICOS de la Ciudad de México. Marzo, 2004

\begin{tabular}{|c|c|c|c|c|c|}
\hline Institución & Lugar & Monitores colocados & Monitores perdidos & Monitores analizados & Monitores < CMD \\
\hline Hospital & Áreas de médicos & 5 & 1 & 4 & 1 \\
\hline \multirow[t]{7}{*}{$(n=1)$} & Áreas de enfermería & 5 & I & 4 & 2 \\
\hline & Baños & 5 & 0 & 5 & 0 \\
\hline & Quirófanos & 3 & I & 2 & 0 \\
\hline & Oficinas & 3 & 0 & 3 & 0 \\
\hline & Cafeterías & 3 & I & 2 & 2 \\
\hline & Escaleras & 3 & 0 & 3 & 0 \\
\hline & Subtotal & 27 & 4 & 23 & 5 \\
\hline Escuelas & Sala de profesores & 2 & 0 & 2 & 0 \\
\hline \multirow[t]{4}{*}{$(n=2)$} & Baños & 4 & 2 & 2 & 2 \\
\hline & Cafeterías & 6 & I & 5 & 5 \\
\hline & Escaleras & 6 & 3 & 3 & 3 \\
\hline & Subtotal & 18 & 6 & 12 & 10 \\
\hline Oficina pública & Oficinas & 10 & 0 & 10 & I \\
\hline \multirow[t]{4}{*}{$(n=1)$} & Atención al público & 5 & I & 4 & 0 \\
\hline & Cafetería & 3 & 0 & 3 & 0 \\
\hline & Escaleras & 3 & 0 & 3 & 0 \\
\hline & Subtotal & 21 & I & 20 & I \\
\hline Aeropuerto & Inmigración & 2 & 0 & 2 & 0 \\
\hline \multirow[t]{5}{*}{$(n=1)$} & Embarque & 3 & 0 & 3 & 0 \\
\hline & Facturación & 3 & 0 & 3 & 0 \\
\hline & Equipaje & 3 & 0 & 3 & 0 \\
\hline & Cafetería & 3 & 0 & 3 & 0 \\
\hline & Subtotal & 14 & 0 & 14 & 0 \\
\hline Restaurantes & Área de fumar & 7 & I & 6 & 0 \\
\hline \multirow[t]{2}{*}{$(n=7)$} & Área de no fumar & 7 & 0 & 7 & 0 \\
\hline & Subtotal & 14 & I & 13 & 0 \\
\hline Bares & Área de fumar & 6 & 0 & 6 & 0 \\
\hline$(n=3)$ & Subtotal & 6 & 0 & 6 & 0 \\
\hline Total $(n=\mid 5)$ & & 100 & 12 & 88 & 16 \\
\hline
\end{tabular}

otros. En particular, los valores más altos de nicotina en el aeropuerto se observaron en el área de fumar de la cafeter a, mientras que en las oficinas públicas las escaleras mostraron las concentraciones más altas. Los restaurantes registraron grados de nicotina consistentemente altos, sin diferencias importantes en las concentraciones de nicotina en las áreas de fumar y no fumar. Los bares presentaron las concentraciones de nicotina más altas y consistentes. La mediana de exposición a humo de tabaco ambiental en bares es 8.7 veces la mediana observada en restaurantes y 545 veces la mediana en hospitales. El grado de variabilidad en aeropuertos y oficinas públicas se evidencia en el número de observaciones extremas o fuera del rango intercuart lico registradas en tales lugares (figura 1).

Los valores de nicotina en las áreas de fumar y no fumar en restaurantes son similares, mientras que en el aeropuerto tienden a ser distintas (cuadro III). En el área 
de fumar de los restaurantes la mediana fue de 0.765 $\mu \mathrm{g} / \mathrm{m}^{3}$ (RIC: $0.545,0.857$ ), y en la de no fumar de $0.684 \mu \mathrm{g} / \mathrm{m}^{3}$ (RIC: $0.471,1.229$ ). En el área de fumar de los aeropuertos la mediana fue de $1.480 \mu \mathrm{g} / \mathrm{m}^{3}$ (RIC: $1.207,1.843)$, y en la de no fumar de $0.076 \mu \mathrm{g} / \mathrm{m}^{3}$ (RIC: $0.041,0.258$ ).

\section{Discusión}

El objetivo de este estudio fue evaluar la presencia y el grado de exposición involuntaria a humo de tabaco en diferentes lugares públicos de la Ciudad de México. En todos los lugares públicos se pudo detectar nicotina, con valores altos en bares, restaurantes y zonas de fumar de los aeropuertos. En escuelas, hospitales y oficinas públicas, los valores de nicotina encontrados se mantuvieron debajo de $0.1 \mu \mathrm{g} / \mathrm{m}^{3}$, y se registró la menor proporción de mediciones por encima de la concentración m nima de detección. Sin embargo, debe considerarse que en las escuelas una tercera parte de los monitores se perdió,

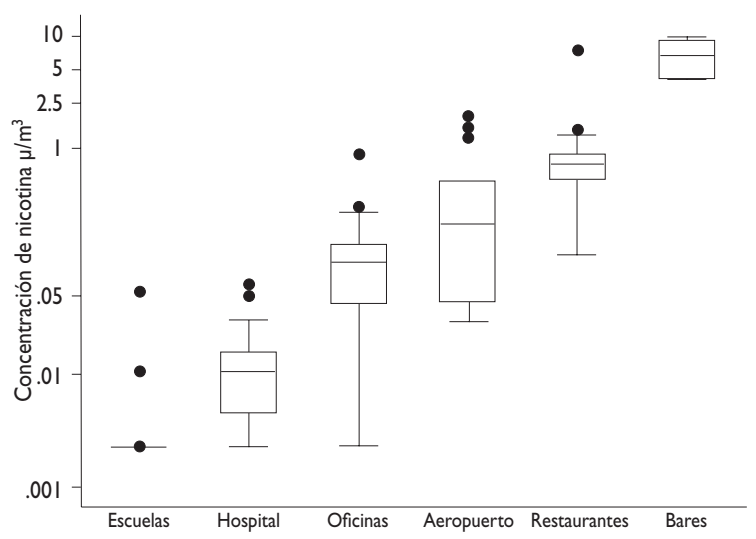

* Las líneas horizontales dentro de los rectángulos representan la mediana; los rectángulos, el rango intercuartil; las barras de error, los valores dentro de 1.5 veces el rango intercuartil; los puntos sólidos, las observaciones atípicas

Figura I. Concentraciones de nicotina ambiental* en lugares públicos de la Ciudad de México. Marzo, 2004

Cuadro II

Medidas de tendencia Central y disPersión de las ConCentraciones de Nicotina AMBiental ( $\mu g / \mathrm{m}^{3}$ ) en lugares públicos de la Ciudad de México. Marzo, 2004

\begin{tabular}{|c|c|c|c|c|c|c|c|}
\hline Lugar & Monitores & Mediana & QI & Q3 & Min & Max & $\%$ Monitores >CMD \\
\hline Hospitales & 23 & 0.011 & 0.005 & 0.159 & $<C M D$ & 0.625 & 82 \\
\hline Escuelas & 12 & $<C M D$ & $<C M D$ & $<C M D$ & $<C M D$ & 0.537 & 45 \\
\hline Oficina pública & 20 & 0.097 & 0.042 & 0.143 & $<C M D$ & 0.864 & 95 \\
\hline Aeropuerto & 14 & 0.207 & 0.043 & 0.498 & 0.028 & 1.843 & 100 \\
\hline Restaurantes & 13 & 0.689 & 0.519 & 0.857 & 0.109 & 6.837 & 100 \\
\hline Bares & 6 & 6.005 & 3.853 & 8.597 & 3.788 & 8.863 & 100 \\
\hline Todos & 88 & 0.058 & 0.010 & 0.384 & 0.002 & 8.863 & 82 \\
\hline
\end{tabular}

QI=Percentil 25, Q3=Percentil 75, Min=Mínimo, Max=Máximo, CMD=Concentración Mínima de Detección

Cuadro III

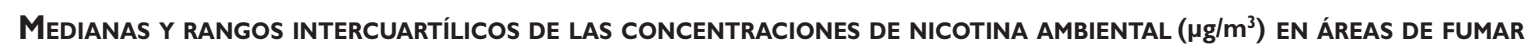
y no fumar de restaurantes y aeropuerto de la Ciudad de México. Marzo 2004

\begin{tabular}{|c|c|c|c|c|c|c|c|}
\hline Lugar & Área & Monitores & Mediana & QI & Q3 & Min & Max \\
\hline \multirow[t]{2}{*}{ Restaurantes } & Fumar & 6 & 0.765 & 0.545 & 0.857 & 0.153 & I.372 \\
\hline & No fumar & 7 & 0.684 & $0.47 I$ & 1.229 & 0.109 & 6.837 \\
\hline \multirow[t]{2}{*}{ Aeropuerto } & Fumar & 3 & 1.480 & 1.207 & 1.843 & 1.206 & 1.843 \\
\hline & No fumar & II & 0.076 & 0.041 & 0.258 & 0.028 & 0.498 \\
\hline
\end{tabular}

*QI=Percentil 25, Q3=Percentil 75, Min=Mínimo, Max=Máximo 
hecho que puede deberse a intentos deliberados de ocultar la exposición. En aeropuertos, restaurantes y bares los valores detectados sobrepasaron $0.1 \mu \mathrm{g} / \mathrm{m}^{3}$, con concentraciones tan altas como $1.843 \mu \mathrm{g} / \mathrm{m}^{3}$ en las áreas de fumar de los aeropuertos y $8.863 \mu \mathrm{g} / \mathrm{m}^{3}$ en los bares. Las zonas de no fumar exhibieron concentraciones menores de nicotina sólo en los aeropuertos, mientras que en los restaurantes las concentraciones de nicotina fueron similares independientemente de la zona, lo que revela la inutilidad de la separación por zonas para controlar la exposición a humo de tabaco ambiental.

El presente estudio utilizó un protocolo común desarrollado por la Escuela de Salud Pública de la Universidad Johns Hopkins y la Organización Panamericana de la Salud. Antes, este protocolo se utilizó en Argentina, Brasil, Chile, Costa Rica, Honduras, Paraguay, Panamá, Perú y Uruguay en un estudio multicéntrico. ${ }^{11,17,18}$ En conjunto, en estos pa ses se detectó nicotina en $94 \%$ de los monitores colocados (rango: 46\% en Panamá a 100\% en Argentina y Uruguay), un porcentaje un poco mayor al de $82 \%$ que se encontró en México (cuadro IV). Las concentraciones de nicotina ambiental que se determinaron en hospitales, oficinas públicas, aeropuertos y restaurantes de México ocupan lugares intermedios en el cuadro de comparación con los demás pa ses latinoamericanos incluidos en el protocolo común. Las concentraciones encontradas en escuelas de México son, junto con la de Costa Rica y Panamá, las más bajas de Latinoamérica. Las concentraciones de nicotina ambiental en bares ocupan el segundo lugar entre las más altas observadas en Latinoamérica, con valores apenas por debajo de los observados en Perú.

El uso de protocolos similares en Europa ${ }^{14}$ y China* permite también la comparación de las concentraciones de nicotina con dichas regiones, salvo en los bares, donde hubo importantes diferencias en los protocolos. Para el resto de lugares públicos, los niveles de nicotina ambiental encontrados en México y en otras ciudades de Latinoamérica fueron menores, por lo general, que las concentraciones que se midieron en la mayor a de pa ses europeos y ciudades chinas. Estas diferencias regionales podr an deberse al menor consumo de cigarrillos por d a en los pa ses de Latinoamérica, diferencias climáticas, diferencias en los sistemas de ventilación de los lugares o en la implementación de la legislación y las normas en cada institución.

Investigaciones previas concluyen que cualquier grado de exposición al humo de tabaco ambiental es potencialmente nocivo. ${ }^{1}$ Para efectos ilustrativos, una aproximación al potencial impacto carcinógeno de la exposición a las concentraciones de humo de tabaco ambiental registradas en este estudio puede derivarse del algoritmo de Repace y Lowry. ${ }^{19}$ De acuerdo con Repace y Lowry, pueden ocurrir 13 decesos por cáncer

Stillman FA, Navas-Acien A, Ma J, Ma S, Avila-Tang E, Breysse P, et
al. Secondhand tobacco smoke in public places in urban and rural China. Tob Control. Documento no publicado.

\section{Concentraciones* de nicotina ambiental en lugares públicos en ciudades Capitales de América Latina. JULIO 2002 A DICIEMBRE 2004. ${ }^{\ddagger 4,11,12}$}

\begin{tabular}{|c|c|c|c|c|c|c|c|c|}
\hline País & & Hospitales & Escuelas & Oficinas Públicas & Aeropuertos & Restaurantes & Bares & $\%$ Monitores $>C M D$ \\
\hline Argentina & 1.33 & $(0.5 I, 3.12)$ & $0.14 \quad(0.05,0.23)$ & $2.60 \quad(1.43,2.99)$ & $0.26(0.18,0.67)$ & $2.04(0.90,2.60)$ & $3.65(3.58,3.65)$ & 100 \\
\hline Brasil & 0.05 & $(0.02,0.09)$ & $<\mathrm{ND}(<\mathrm{ND}, 0.03)$ & $0.04 \quad(0.03,0.21)$ & $0.10(0.03,0.16)$ & $2.52^{\S}(1.25,3.95)$ & & 83 \\
\hline Chile & 0.13 & $(0.03,0.47)$ & $0.01 \quad(0.003,0.02)$ & $1.13 \quad(0.08,0.13)$ & $\mathrm{I} .07(0.64, \mathrm{I} .07)$ & $2.08(1.08,3.56)$ & $3.33(2.06,4.82)$ & 97 \\
\hline Costa Rica & 0.02 & $(0.01,0.04)$ & $<\mathrm{ND}(<\mathrm{ND},<\mathrm{ND})$ & $0.02(0.01,0.05)$ & $0.01(0.01,0.03)$ & $0.73(0.46,1.80)$ & $1.32(0.75,7.43)$ & 90 \\
\hline Honduras & $<N D$ & $(<N D, 0.02)$ & $0.003(<N D, 0.005)$ & $0.05 \quad(0.01,0.15)$ & $0.07(0.02,0.59)$ & $\mathrm{I} .68(0.35,2.29)$ & $1.26(0.77,1.77)$ & 82 \\
\hline México & 0.01 & $(0.005-0.16)$ & $<N \quad(<N D,<N D)$ & $0.10(0.04-0.14)$ & $0.21(0.04-0.50)$ & $0.69(0.52-0.86)$ & $6.00(3.85-8.60)$ & 82 \\
\hline Panamá & $<N D$ & $(<\mathrm{ND},<\mathrm{ND})$ & $<\mathrm{ND}(<\mathrm{ND},<\mathrm{ND})$ & $<\mathrm{ND}(<\mathrm{ND}, 0.004)$ & $0.03(0.01,0.69)$ & $0.15 \quad(0.01,2.10)$ & $2.36(2.14,2.88)$ & 46 \\
\hline Paraguay & 0.01 & $(0.001,0.03)$ & $0.001(<N D, 0.05)$ & $0.05 \quad(0.03,0.10)$ & $0.07(0.05,0.20)$ & $0.24 \quad(0.06,0,36)$ & $1.59(0.78,6.68)$ & 87 \\
\hline Perú & 0.02 & $(0.01,0.07)$ & $0.01 \quad(0.01,0.08)$ & $0.07 \quad(0.01,0.31)$ & $0.93(0.20,1.30)$ & $(0.15,2.42)$ & $6.21(4.00,9.12)$ & 97 \\
\hline Uruguay & 0.80 & $(0.31,1.69)$ & $0.08 \quad(0.02,0.61)$ & $0.67 \quad(0.47,0.94)$ & $1.15(0.74,2.49)$ & I.4I $\quad(0.4 I, 2.48)$ & $3.14(0.77,4.82)$ & 100 \\
\hline $\begin{aligned} & * \text { Mediana ( } \\
& \text { Monitore } \\
& \text { (diciembr } \\
& \text { CMD y N } \\
& \S \text { En Brasil }\end{aligned}$ & $\begin{array}{l}\text { Ingo in } \\
\text { efectu } \\
\text { de } 200 \\
\text { : Conc }\end{array}$ & $\begin{array}{l}\text { ntercuartílico) } \\
\text { uado de julio de } \\
\text { 04) }\end{array}$ & 02 a febrero de 20 & en todos los país & excepto en Ho & $s$ (octubre de & Mávico (mo & de 2004) y Panamá \\
\hline
\end{tabular}


de pulmón en cada 100000 personas expuestas a un microgramo de nicotina por metro cúbico durante 40 años. As, las concentraciones observadas en bares y restaurantes se traducir an, respectivamente, en $80 \mathrm{y}$ 9 decesos por cada 100000 personas expuestas a un microgramo de nicotina por metro cúbico durante 40 años. Debe recalcarse que los números obtenidos con el algoritmo sólo son ilustrativos del impacto potencial que la exposición podr a tener y no una cuantificación directa del riesgo. Asimismo, ese estimado considera de manera exclusiva las muertes por cáncer de pulmón, sin incluir la mortalidad o morbilidad derivada de otros padecimientos vinculados con el humo de tabaco.

Algunas limitaciones del presente estudio merecen discutirse. La selección de los lugares por conveniencia para el monitoreo podr a reducir la generalización al resto de los lugares de la Ciudad de México. Sin embargo, el presente estudio fue desarrollado para cuantificar los valores de nicotina ambiental y subsanar la ausencia de información sobre exposición involuntaria al humo de tabaco en la Ciudad de México, sin la pretensión de obtener un estimado representativo. La documentación de la presencia de nicotina en los lugares públicos muestreados en este estudio es útil para diseñar futuras evaluaciones y mantener un sistema de vigilancia de exposición al humo de tabaco. Más importante, esta primera evaluación del grado de apego a la legislación vigente encargada de controlar el consumo de tabaco en los lugares públicos indica que la implementación de las leyes debe ser reforzada en ciertos lugares como oficinas públicas y el aeropuerto, y que legislación adicional es necesaria para reducir y eliminar la exposición al humo de tabaco en bares y restaurantes. Por razones log sticas, se utilizó un monitoreo continuo de 24 horas, todos los d as, por una o dos semanas. En este caso, el monitoreo continuo tiende a subestimar la exposición, ya que la mayor parte de los lugares evaluados no funciona 24 horas continuas ni todos los d as de la semana. Sin embargo, a pesar de la subestimación, los valores registrados son muy altos en bares, restaurantes y áreas de fumar en aeropuertos.

Este estudio representa la primera aproximación sistemática a la medición del humo de tabaco en lugares públicos en México. Los lugares monitoreados incluyeron el tipo de espacios protegidos por la legislación mexicana, además de bares y restaurantes. El protocolo común utilizado y aplicado antes en otros pa ses de Latinoamérica facilitó la comparación de los valores registrados en México respecto a otros pa ses de la región. El uso de protocolos similares en Europa y China también permite la comparación con estas regiones.

Los resultados del presente estudio ponen de manifiesto la falta de cumplimiento con las regulacio- nes federales sobre el consumo de tabaco en lugares públicos. Particular atención merecen los bares, restaurantes, aeropuertos y oficinas públicas, donde se registraron las concentraciones de nicotina más altas. En los restaurantes, la división en áreas de fumar y no fumar no es efectiva para disminuir los grados de exposición al humo de tabaco, por lo que es evidente la necesidad de eliminar por completo el consumo de tabaco en los mismos. Las escuelas y los hospitales registraron niveles menores; sin embargo, se detectó nicotina en varias de las muestras, por lo que aún no pueden considerarse espacios $100 \%$ libres de humo de tabaco. Deben efectuarse esfuerzos adicionales para asegurar espacios libres de tabaco en aquellos lugares protegidos por la legislación mexicana, incluyendo un sistema de vigilancia continua de exposición al humo de tabaco ambiental. En México, el Instituto Nacional de Salud Pública cuenta con el Laboratorio Anal tico de Compuestos del Tabaco (http:/ / www.insp.mx/Portal/lacot/lacot03.html), un recurso fundamental para facilitar la vigilancia epidemiológica de la exposición al humo de tabaco ambiental. La exposición al humo de tabaco ambiental en los lugares públicos no sólo afecta a los usuarios sino también a los trabajadores, quienes suelen estar expuestos por una mayor cantidad de tiempo. Con el fin de proteger a usuarios y trabajadores de todos los lugares públicos, es fundamental promover una legislación que garantice espacios libres de tabaco en todos los lugares públicos y centros de trabajo. Será importante que futuras investigaciones evalúen con profundidad el éxito de la legislación sobre los espacios libres de humo de tabaco, en particular en bares y restaurantes.

\section{Agradecimientos}

Tonatiuh Barrientos-Gutiérrez ha contado con una beca Fogarty International Center Training Grant (3 D43 TW00644). La doctora Ana Navas-Acien cuenta con apoyo del Instituto de Investigación Médica de Asistentes de Vuelo (Flight Attendant Medical Research Institute). La Organización Panamericana de la Salud organizó el entrenamiento para la realización del estudio. Los análisis de laboratorio se realizaron en el Center for Urban Environmental Health, Johns Hopkins Bloomberg School of Public Health, bajo la dirección del doctor Breysse. Agradecemos a los doctores David Gimeno, Keith Bureau y Elaine Symanski sus sugerencias para el análisis de los datos. Agradecemos a Marisol Acuña (Ministerio de Salud, Santiago, Chile), Marta Angueira (UATA, Argentina), Adriana Blanco-Marquizo (Intendencia Municipal de Montevideo, Uruguay), Carmen Barco (CEDRO, Perú), Vera L. Colombo (INCA, R o de Janeiro, Brasil), 
Graciela Gamarra (Ministerio de Salud, Paraguay), Claudia D. Gómez (IHADFA, Tegucigalpa, Honduras), Katya Jiménez-Reyes (IAFA, San José, Costa Rica), Reina Roa (Ministerio de Salud, Panamá) y Alfonso Zavaleta (CEDRO, Perú) su contribución para la comparación con otros pa ses de Latinoamérica.

\section{Referencias}

I. US Department of Health and Human Services. The health consequences of involuntary exposure to tobacco smoke:A report of the Surgeon General. Atlanta, GA, USA, 2006. Disponible en: http://www.surgeongeneral.gov/library/secondhandsmoke/ 2.World Health Organization. WHO Framework Convention on Tobacco Control. Geneva:WHO, 2003. Disponible en: http://www.who.int/tobacco/framework/en/

3. Goodman P,Agnew M, McCaffrey M, Paul G, Clancy L. Effects of the Irish smoking bar on respiratory health of bar workers and air quality in Dublin pubs. Am J Respir Crit Care Med 2007;75(8):799-750. 4. Gallus S, Zuccaro P, Colombo P,Apolone G, Pacifici R, Garattini S, et al. Effects of new smoking regulations in Italy.Ann Oncol 2006; 17:346-347. 5. Moskowitz J, Lin Z, Hudes E. The impact of workplace smoking ordinances in California on smoking cessation. Am J Public Health 2000;90:757-76I.

6. Abrams SM, Mahoney MC, Hyland A, Cummings KM, Davis W, Song L. Early evidence on the effectiveness of clean indoor air legislation in New York State. Am J Public Health 2006;96:296-298.

7. Ministerio de Salud Pública. Establecimiento de áreas de fumadores en restaurantes, bares y lugares de esparcimiento. Montevideo, Uruguay, 2005; I68/005. Disponible en: http://www.presidencia.gub.uy/_web/ decretos/2005/05/39_05\%2004\%202005_0000I.PDF.

8. Legislatura de Puerto Rico. Ley para reglamentar la práctica de fumar en lugares públicos. San Juan, Puerto Rico, 2006; 66-2073. Disponible en: http://www.pulmon.org/Ley_Num_66.htm.
9. Legislatura de la Ciudad Autónoma de Buenos Aires. Ley de Control del Tabaco.Argentina: Ciudad Autónoma de Buenos Aires, 2005; 1799. Disponible en: http://www.cedom.gov.ar/es/legislacion/normas/leyes/html/ leyl799.html.

I0. Ibáñez-Hernández N. Disposiciones jurídicas sobre la protección de los no fumadores en los estados de la República Mexicana. En: Primer Informe Sobre El Combate Al Tabaquismo. México Ante El Convenio Marco Para El Control Del Tabaco. Cuernavaca, México: Instituto Nacional de Salud Pública, 2005:149-I59.

II. Navas-Acien A, Peruga A, Breysse P, Zavaleta A, Blanco-Marquizo A, Pitarque R, et al. Secondhand tobacco smoke in public places in Latin America, 2002-2003. JAMA 2004;291:2741-2745.

12. Hammond SK, Leaderer BP.A diffusion monitor to measure exposure to passive smoking. Environ Sci Technol 1987;21:494-497.

13. Ogden MW, Maiolo KC. Comparative evaluation of diffusive and active sampling systems for determining airborne nicotine and 3-ethenylpyridine. Environ Sci Technol 1992;26:1226-I 234.

14. Nebot M, López MJ, Gorini G, Neuberger M,Axelsson S, Pilali S, et al. Environmental tobacco smoke exposure in public places of European cities. Tob Control 2005; 14:60-63.

I5. Mulcahy M, Evans DS, Hammond SK, Repace JL, Byrne M. Secondhand smoke exposure and risk following the Irish smoking ban: an assessment of salivary cotinine concentrations in hotel workers and air nicotine levels in bars. Tob Control 2005; | 4:384-388.

16. Schorp MK, Leyden DE. Distribution analysis of airborne nicotine concentrations in hospitality facilities. Environ Int 2002;27:567-578.

17. Navas-Acien A, Gómez-Mass CD, Durón HV,Alvarenga-Chinchilla E. Exposición ambiental a humo de tabaco en Tegucigalpa, Honduras. Rev Fac Ciencias Médicas Hondureña 2005;2:5I-59.

18. Roa R, Luque H, Holder K, Chang A, Gonzalez I, Navas-Acien A, et al. Estudio de vigilancia de humo ambiental de tabaco. Panamá: Ciudad de Panamá. Serie Comunicación Científica del Ministerio de Salud, 2004; Vol. 2. Disponible en: http://ops-oms.org.pa/docs/tabaco.pdf.

19. Repace JL, Lowrey AH.An enforceable indoor air quality standard for environmental tobacco smoke in the workplace. Risk Anal 1993; 13:463-475. 